\title{
Sistemas agroforestales de café en Veracruz, México: identificación y cuantificación espacial usando SIG, percepción remota y conocimiento local Coffee agroforestry systems in Veracruz, Mexico: spatial identification and quantification using GIS, remote sensing and local knowledge
}

\author{
Eva Aurora Bautista Calderon ${ }^{1}$, Edgar Vladimir Gutiérrez Castorena ${ }^{2 *}$, \\ Víctor Manuel Ordaz Chaparro ${ }^{1}$, Ma. del Carmen Gutiérrez Castorena ${ }^{1}$ y Lenom Cajuste Bontemps ${ }^{1}$ \\ ${ }^{1}$ Colegio de Postgraduados, Campus Montecillo. Carretera México-Texcoco km 36.5. 56230 Montecillo, Texcoco, Estado de México, México. \\ ${ }^{2}$ Facultad de Agronomía, Universidad Autónoma de Nuevo León. Calle Francisco Villa S/N, Ex Hacienda el Canadá. General Escobedo, N.L., México. \\ *Autor responsable (edgar.gutierrezcs@uanl.edu.mx)
}

\section{RESUMEN}

La región montañosa de Veracruz es un ecosistema complejo por su fisiografía y vegetación natural en donde ocurren sistemas productivos agroforestales de café que son de alto valor económico para los productores. Estos sistemas presentan múltiples factores bióticos y abióticos que dificultan su zonificación y clasificación y por ende está limitada la transferencia de tecnología entre productores y técnicos. El objetivo de la presente investigación fue generar mapas temáticos de la distribución y aptitud de la tierra con potencial productivo de cultivo de café en los sistemas agroforestales de la zona montañosa del municipio de Huatusco, Veracruz. Para ello se emplearon técnicas de análisis de percepción remota (PR), con enfoque paramétrico sobre Sistemas de Información Geográfica (GIS), clasificación interpretativa de tierras y conocimiento local. En la zona de estudio se presentan dos componentes agroforestales: bosque mesófilo de montaña (BMM) $(27 \%)$ y potreros $(\mathrm{Po})(16 \%)$ y tres subcomponentes: policultivo tradicional $(\mathrm{Pt})$ y comercial $(\mathrm{Pc})(36 \%)$, monocultivo especializado (Me) $(3 \%)$ y zonas urbanas (Zu) $(2.9 \%)$, identificados a través de su brillantez y textura en una imagen de satélite; sin embargo, hay confusión entre la vegetación nativa y comercial. Con la participación de los productores, estos componentes se identificaron con una precisión de $78.9 \%$ (Pc),

Cita recomendada:

Bautista Calderon, E. A., E. V. Gutiérrez Castorena, V. M. Ordaz Chaparro, Ma. del C. Gutiérrez Castorena y L. Cajuste Bontemps. 2018. Sistemas agroforestales de café en Veracruz, México: identificación y cuantificación espacial usando SIG, percepción remota y conocimiento local. Terra Latinoamericana 36: 261-273.

DOI: https://doi.org/10.28940/terra.v36i3.350
$87.5 \%$ (Pt) y 100\% (BMM, Pot, Me). La zona de mayor aptitud para la producción de café se localiza en la zona centro (23\%); mientras que los de baja calidad en el centro y noreste $(45.5 \%)$ del municipio. Los BMM ocurren en áreas no aptas para la producción de cultivo de café. La combinación entre técnicas geoespaciales con el conocimiento local de la aptitud de las tierras permite identificar zonas con potencial productivo agrícola de café y dar elementos para la conservación de otros ambientes como el BMM.

Palabras clave: clasificación interpretativa, análisis de imágenes de satélite, spot 5, bosques mesófilos de montaña.

\section{SUMMARY}

The mountainous region of Veracruz is a complex ecosystem due to its physiography and natural vegetation where agroforestry coffee production systems occur that are of high economic value for producers. These systems have multiple biotic and abiotic factors that hinder their zoning and classification, and therefore, the transfer of technology between producers and technicians is limited. The objective of the present investigation was to generate thematic maps of the distribution and aptitude of the land with productive potential for coffee cultivation in the agroforestry systems of the mountainous area 
of the municipality of Huatusco, Veracruz. For this, remote perception analysis (PR) techniques were used, with a parametric approach on Geographic Information Systems (GIS), interpretative land classification and local knowledge. Two agroforestry components are presented in the study area: mountain mesophilic forest (BMM) (27\%) and paddock (Pot) (16\%) and three subcomponents: traditional polyculture $(\mathrm{Pt})$, commercial polyculture (Pc) (36\%), specialized monoculture $(\mathrm{Me})(3 \%)$ and urban areas $(\mathrm{Zu})(2.9 \%)$, identified by their brilliance and texture in a satellite image; however, there is confusion between native and commercial vegetation. With the participation of the producers, these components were identified with an accuracy of $78.9 \%(\mathrm{Pc}), 87.5 \%(\mathrm{Pt})$ and $100 \%$ (BMM, Pot, Me). The area of greatest aptitude for coffee production is located in the central zone $(23 \%)$; while those of low quality are in the center and northeast $(45.5 \%)$ of the municipality. BMMs occur in areas not suitable for coffee production. The combination of geospatial techniques with local knowledge of land aptitude allows identification of areas with agricultural potential for coffee production, providing elements for conservation of other environments such as the BMM.

Index words: interpretative classification, analysis of satellite images, spot 5, mesophilic mountain forests.

\section{INTRODUCCIÓN}

Los Sistemas de Información Geográfico (SIG) trabajan con datos espaciales del mundo físico para generar mapas temáticos (Brody, 1999), para lo cual se realiza captura, almacenamiento y despliegue de información mediante diferentes procesos operacionales (Rosete y Bocco, 2003). El manejo de grandes volúmenes de datos y organización de la información (Instituto de Investigación de Recursos Biológicos Alexander Von Humboldt, 2006) se convierte en una herramienta útil en la toma de decisiones (Gómez et al., 2003).

Los SIG principalmente se utilizan para evaluar coberturas vegetales de ecosistemas (Orradre, 2014) inventarios forestales (Gutiérrez y Gould, 1994) y dinámica del medio biótico y abiótico (Bermejo et al., 2005) ${ }^{1}$. Además, los metadatos del SIG se pueden combinar con imágenes de satélite (García, 2006) para evaluar el manejo de los agroecosistemas con base en cambios temporales (Buendía et al., 2002). La información digital en cada una de sus bandas de la imagen de satélite puede ser manipulada para agrupar objetos con diferentes tamaños, formas y colores. Asimismo, se puede incorporar criterios más complejos como textura, brillo, tamaño o combinación entre ellos (Chuvieco, 2008). Esta combinación permite al intérprete rapidez y confiabilidad en la extracción cartográfica de los recursos naturales (a corto, mediano y largo plazo) con determinada periodicidad (Rullán et al., 2011). A pesar de ello, García (2006) indica que muchos de los objetos no suelen ser clasificados de forma correcta, dado que depende de la experiencia y percepción individual del técnico. También, es común que los estudios se realicen principalmente en gabinete con pocos o nulos recorridos en campo sin verificación de linderos.

Otra técnica de conceptualización de la realidad física es mediante la clasificación interpretativa, la cual identifica objetos visibles sobre la imagen que posteriormente los representa cartográficamente. La clasificación de tierras agrícolas con diversidad de cultivos (usos/cobertura) (Cayssials y Alvarez, 1983), elaboración de una calificación biológica de la vegetación y usos de suelo (Vega et al., 2008), evaluación de cobertura y uso de la tierra (Zorogastúa et al., 2011), se han llevado a cabo utilizando esta metodología. Además, esta técnica ha sido muy útil en estudios sobre diversificación forestal de bosques andinos (Lencinas, 2011), delimitación y detección de áreas con alta probabilidad de incendios forestales (Verdú y Salas, 2010), o en combinación con procesos digitales-analógicos en imágenes de satélite (Portuguez, 1998).

Por otra parte, el análisis digital de imágenes y su representación en mapas temáticos está directamente relacionado con enfoques paramétricos, que consideran un clasificador por cada componente, por ejemplo, suelos (Borrás et al., 2017), temperatura, vegetación; o en la clasificación de las tierras con base en sus características físicas, químicas o estructurales (Ortiz y Cuanalo, 1978). No obstante, a pesar de las ventajas particulares, estas técnicas individuales o en combinación están limitadas para expresar divergencias

\footnotetext{
${ }^{1}$ Bermejo D., J. A., P. L. Pérez de la Paz y M. J. Del Arco Aguilar. 2005. Aplicación de los sistemas de información geográfica (SIG) en el análisis predictivo de flora en peligro de extinción: Lotus eremiticus. Santos (Isla de la Palma - Islas Canarias). pp. 271-274. In: XI Congreso Nacional de Teledetección. Puerto de la Cruz, Tenerife, España.
} 
entre objetos que comparten las mismas características. En el caso de sistemas agroforestales de café se ha reportado que se pueden confundir con los ecosistemas forestales o de selva ya sea que estén bajo sombra o incluso sin cobertura arbórea (Escamilla y Díaz, 2002), por lo que se tienen que realizar estudios directamente en campo con apoyo de los productores.

El café es un arbusto de hoja perenne globosa, de tallo múltiple o árbol pequeño cuando está podado que se intercala con diferentes tipos de vegetación (ECOCROP, 2018) y es principalmente de secano por las condiciones climáticas en donde se desarrolla. Es una planta que tiene un alto valor de interés económico para nuestro país ya que somos los principales productores de café orgánico del mundo (La Jornada, $2018)^{2}$. Este agroecosistema tiene enorme relevancia ecológica (Escamilla et al., 2005), por la gran diversidad de especies (Moguel y Toledo, 2004; Perfecto et al., 2005). Además, es necesario ubicar las zonas de alta calidad para la producción de café, pero sin afectar a los bosques mesófilos de montaña que están siendo presionados por el cambio de uso del suelo (Martínez et al., 2009) y es ahí donde el conocimiento local es relevante (Reyes-García, 2009) sobre todo para la trasferencia del manejo agronómico. La hipótesis que se plantea es que los sistemas agroforestales de café insertos en zonas montañosas con alta diversidad y complejidad de estructuras y manejo agrícola se pueden zonificar y cuantificar a través de la técnica de clasificación interpretativa en combinación con SIG, análisis de imágenes y conocimiento local de tierras para determinar las zonas de alto potencial del cultivo, para lo cual el objetivo que se planteó fue generar mapas temáticos de la distribución y aptitud potencial de cultivo de café en los sistemas agroforestales de la zona montañosa del municipio de Huatusco, Veracruz.

\section{MATERIALES Y MÉTODOS}

\section{Área de Estudio}

El municipio de Huatusco de Cuellar pertenece a la región de "Las Montañas" y se ubica en la zona centro del estado de Veracruz, entre las coordenadas $19^{\circ} 04^{\prime}$ y $19^{\circ} 13^{\prime} \mathrm{N}$ y los meridianos $96^{\circ} 41^{\prime}$ y $97^{\circ}$ 04' O, con una superficie de 19995 ha (Figura 1).

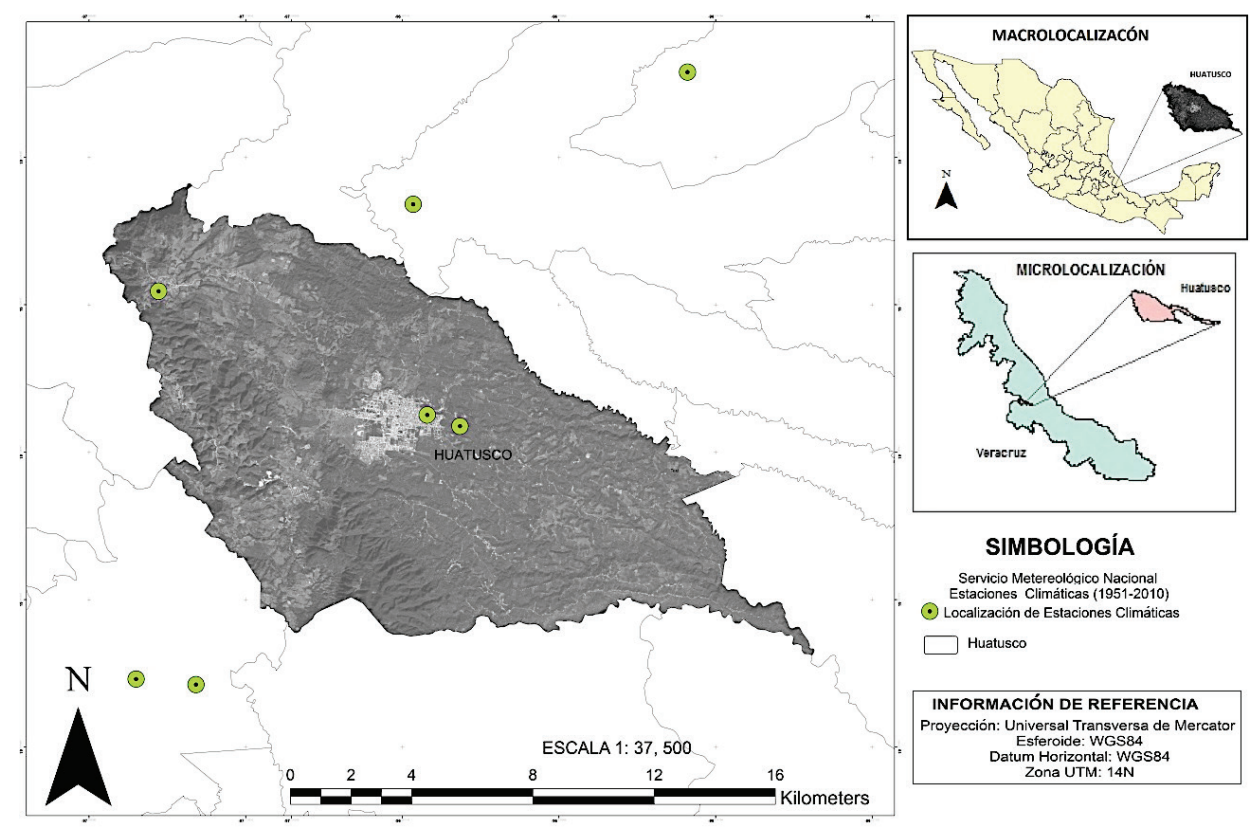

Figura 1. Localización de la zona de estudio (Huatusco de Cuellar, Veracruz, México) y localización de estaciones meteorológicas.

${ }^{2}$ La Jornada Periódico. 2018. Publicado y consultado el 21 de mayo de 2018 en: http://www.jornada.com.mx/ultimas/2018/05/21/mexico-principal-productorde-cafes-organicos-en-el-mundo-6058.html. (Consulta: mayo 25, 2018). 
El relieve es montañoso de norte a sur y pertenece al eje Neo-volcánico o Sistema Tarasco Nahua; las líneas de crestas se mantienen por arriba de los $2200 \mathrm{msnm}$ (INEGI, 2009a) y se localizan en la región hidrológica Papaloapan en 96\% (INEGI, 2009b). La variabilidad climática de las montañas está representada por seis tipos, de los cuales el Semicálido subhúmedo y el Templado húmedo (INEGI, 2009a) cubren 57 y 20\% respectivamente. La precipitación promedio anual oscila entre 1700 y 2000 mm (SMN, 2017).

\section{Trabajo de Gabinete y Campo}

La presente investigación se llevó a cabo en tres etapas, las cuales se describen a continuación:

1) Pre-campo. Fotointerpretación para delimitar los patrones de vegetación con apariencia semejante en los sistemas agroforestales (escala 1:50 000, intensidad media) sobre la imagen digital (SPOT 5) y selección de campos de entrenamiento (Bolstad, 2012). Elaboración de un levantamiento fisiográfico utilizando modelos de elevación digital del terreno, cartas edafológicas, usos de suelo y vegetación publicadas a escala 1:50 000 (INEGI, 2009a). Además, se realizó el mapa base para esquematizar los tipos de cobertura.

2) De-campo. Reconocimiento en campo de los patrones de vegetación en los sitios de entrenamiento, georreferenciación sobre la imagen, y descripción de características como tono, brillantez, textura, sombras y aspecto de temporalidad (García, 2006). Entrevistas a productores sobre la aptitud de las tierras para la producción de café y verificación y delimitación de linderos de cada componente.

3) Post-campo. Extracción de polígonos de verificación de coberturas a partir del conjunto de información (bases de datos, entrevistas y mapas temáticos); trasferencia de unidades de fotointerpretación a unidades cartográficas; y corrección sobre las unidades cartográficas digitales utilizando una área mínima cartografía (AMC) de $1 \mathrm{~cm}^{2}$ a escala 1:50 000 (Ortiz y Cuanalo, 1978). Posteriormente, los suelos categorizados por su aptitud fueron delimitados en unidades cartográficas utilizando los programas $\operatorname{ArcGis}{ }^{\circledR}$ v10.1 y ERDAS ${ }^{\circledR} \mathrm{v} 10$ sobre la imagen de satélite. La cartografía finalizó con la identificación de los componentes y subcomponentes de los sistemas agroforestales y la elaboración del mapa digital temático de los agroecosistemas de café.

\section{Elaboración de Mapas Temáticos}

La imagen de satélite utilizada tiene una resolución espacial de $20 \times 20 \mathrm{~m}$ por pixel, profundidad de análisis de 8 Bits, georreferenciada a proyección Universal Transversa de Mercator (UTM) esferoide WGS84 zona 14 norte, con metadatos homogeneizados cartográficamente (Guerra y Ochoa, 2005), con nula nubosidad. La imagen fue proporcionada por la Estación de Recepción México de la Constelación SPOT 5 (ERMEXS) (s5_593312_20081221_3a_J ortorrectificada) bajo el convenio ERMEXSSAGARPA.

El mapa temático de los agroecosistemas de café (enfoque paisajista) para evaluar la aptitud potencial del cultivo se realizó utilizando datos paramétricos de pendiente (INEGI, 2009a), temperatura y precipitación (SMN, 2017), el conocimiento local de tierras, algebra de mapas y validación interpretativa. Todos los mapas fueron evaluados a través de valores binarios, donde el "cero" representa las clases con baja calidad o "No Apta" y el "uno" la clase de alta calidad o "Apta" según sus características particulares para la producción agrícola del café. A continuación, se describe cada mapa temático simple y compuesto:

1. Mapa de la aptitud de suelo a través del conocimiento local de tierras. Estos mapas fueron elaborados con la participación e identificación directa en campo por los productores. Los recorridos y entrevistas a productores se realizaron durante ocho días y se establecieron sin remuneración económica de acuerdo con la metodología de Ortiz y Gutiérrez (1999). El cuestionario que se aplicó consideró los siguientes tópicos: variedad de café establecido, arreglo espacial del sembradío, nombre común de especies arbóreas empleadas como sombreaderos, destino de las especies maderables, propósito de las especies asociadas al cultivo de café, calidad de las plantaciones por su producción, motivo donde se encuentran las plantaciones, y razón de su ausencia en la zona norte, calidad de grano obtenido en el predio y causas de baja y alta calidad del grano. La encuesta finalizó cuando la información fue repetitiva.

2. Mapa de pendiente. Este mapa se realizó utilizando al Manual de Conservación del Suelo y Agua (Anaya, 1991) y se digitalizó mediante el módulo "Surface" y el modelo de "Elevación Digital" de ArcGIS ${ }^{\circledR}$ v10. Se obtuvieron ocho clases por grado de pendiente 
$(0-2,2-6,6-10,10-15,15-25,25-40,40-100$ y > 100\%). Aunque todas ellas son clasificadas como "Aptas" en este trabajo se consideraron sólo las primeras cinco clases, es decir pendientes de menos de $40 \%$.

3. Mapas de temperatura y precipitación. Estos mapas fueron generados a partir de la base de datos de 18 estaciones de las Normales Climatológicas (1951-2010) (SMN, 2017) disponible en la red del sitio oficial en formato digital y el modelo de interpolación de Kriging simple (ArcGis ${ }^{\circledR} v 10.1$ ). Las temperaturas medias mensuales del área de estudio fueron categorizadas en tres clases: $<19{ }^{\circ} \mathrm{C}$, alta calidad (ac), entre 19 y $22{ }^{\circ} \mathrm{C}$, mediana calidad (mc), y $>22{ }^{\circ} \mathrm{C}$ baja calidad (bc), de acuerdo con lo que indica Pérez et al. (2005) para la producción de café en los sistemas agroforestales. La precipitación media mensual se categorizó como "Apta" cuando se presentó una precipitación entre 1400 y $2000 \mathrm{~mm}$, intervalo que corresponde a los requerimientos hídricos del cultivo reportados por López-Lara et al. (1997) y Benegas $(2009)^{3}$. Los datos de precipitación fueron obtenidos de las estaciones climáticas reportadas en las normales climáticas (1951-2010) (SMN, 2017).

4) Mapa temático compuesto (vectorial) de agrosistemas de café. Este mapa fue construido mediante la sobre-posición (algebra de mapas) de los mapas de suelo por aptitud de la tierra, pendiente, temperatura y precipitación mediante geoprocesing en ArcGis ${ }^{\circledR} \mathrm{V} 10.1$. Este operador espacial permite la unión de dos o más mapas vectoriales (por producto entre valores binarios) para crear una nueva capa vectorial por atributo. Posteriormente, se seleccionaron los centroides de cada polígono, y se establecieron los campos de entrenamiento

\section{Validación de la Clasificación Interpretativa y Matrices de Confusión}

La validación de los mapas se llevó a cabo con apoyo de técnicos de la Coordinación de Fomento Agropecuario, y su comparación sobre el mapa temático de cobertura. Durante los recorridos se modificaron los linderos entre componentes y subcomponentes, al implementar estrategias de verificación y dividir la zona en cuatro cuadrantes bajo el criterio de accesibilidad a sitios. En cada campo de entrenamiento se confirmaron los linderos de los sistemas agroforestales y se registró el tipo de vegetación nativa y comercial utilizada como sombreaderos por cada tipo de sistema agroforestal. Para llegar a cada sitio se utilizaron como acceso las vías terrestres de comunicación (carreteras y brechas). Finalmente, se realizó una clasificación interpretativa con todos los componentes y se evaluó la concordancia (precisión) entre el usuario y el productor utilizando matrices de confusión de acuerdo con Cohen (1960). En este índice los intervalos varían de -1 a +1 , donde +1 indica una perfecta concordancia y valores a cero o menos indica que la relación es prácticamente al azar.

\section{RESULTADOS Y DISCUSIÓN}

\section{Separabilidad y Fotointerpretación sobre Imagen de Satélite}

Con la fotointerpretación y clasificación interpretativa sobre la imagen de satélite se diferenciaron cuatro componentes de igual nivel jerárquico: a) zona urbana $(\mathrm{Zu})$, b) bosque mesófilo de montaña (BMM), c) potreros (Pot) y d) agricultura (Agr). Los primeros tres componentes se caracterizaron mediante su firma espectral (textura, brillantez y tonalidad) sobre la imagen satelital en ERDAS $\AA_{\mathrm{V}} 10$.

En el caso del componente "Agricultura" se encontró que existe confusión con estructuras vegetativas nativas o con la vegetación comercial, las cuales se utilizan como sombreaderos para el manejo del cultivo de café (Moguel y Toledo, 2004). Es por esa razón que este componente se subdividió en cuatro subcomponentes: a) policultivo tradicional (PT), constituido por especies vegetales nativas o inducidas en plantaciones de café bajo sombra, b) monocultivo especializado (ME) con especie vegetal del genero Inga o cultivo semi-sombreado, c) policultivo comercial (PC) y d) agricultura de grano o vegetales (AgrG) (Cuadro1). Las coberturas vegetales en los subcomponentes fueron identificadas a través de sus firmas espectrales (brillantez y tonalidad), los cuales mostraron una vegetación vigorosa semejante al componente BMM y estructuras en las bandas visibles del espectro electromagnético azul y rojo tal como han sido reportadas por Chuvieco (2008).

\footnotetext{
${ }^{3}$ Benegas, K. 2009. Identificación de las fuentes de variación que tienen efecto sobre la calidad del café (Coffea arabica) en los municipios de el Paraíso y Alauca, Honduras. Tesis Mag. Sc. Turrialba, CR, CATIE. 58 p.
} 
Los mapas temáticos de componentes de BMM, Pot y AgrG no presentaron problemas de separabilidad entre objetos visibles sobre la imagen digital. Los subcomponentes PT, PC y ME fueron semejantes en la mayoría de los campos de capacitación (entrenamiento) y verificación en la tonalidad de gris oscuro, siendo necesario la combinación entre bandas 1, 2, 3 de la imagen (Spot 5) y procesos de análisis de imagen en falso color, textura, forma y tamaño para su separabilidad.

Los resultados del análisis a los subcomponentes en falso color (combinación de bandas $3,2,1$ ) y su separabilidad se produjo en color rojo intenso (entre 155 - 200 valores de pixel en RGB) para vegetación densa en BMM y rojo de menor intensidad (entre 75 - 150 valores de pixel) en coberturas PT, PC y ME. También la textura (rugosidad y suavidad) fue importante para la separabilidad de los componentes; por ejemplo, alta heterogeneidad a nivel de gris y textura rugosa en BMM, texturas lisas y homogéneas en Pot y AgrG y de baja rugosidad y mediana suavidad en los subcomponentes PT, PC, y ME. Estas diferencias en texturas facilitaron su separabilidad tal como ha sido señalado por García (2006) y Chuvieco (2008).

Por otra parte, los procesos de identificación de objetos por "forma" fortaleció la divergencia entre el subcomponente ME contra PT y PC, no así entre los componentes BMM contra PT y PC debido a las características de semejanza por manejo del cultivo.
Por último, el proceso por "tamaño" fue limitado para separar áreas debido a la resolución espacial de la imagen de satélite.

El mapa temático de cobertura y distribución espacial realizado a partir del levantamiento fisiográfico con enfoque paisajista para los componentes ZU, BMM y Pot como para los subcomponentes PT, PC, ME y AgrG se muestran en la Figura 2; mientras que en el Cuadro 1 se reportan las áreas respectivas. La superficie cuantificada con base en la fotointerpretación, clasificación interpretativa y análisis de imágenes para los componentes ZU, BMM y Pot fueron de 3, 22 y $10 \%$ respectivamente; mientras que para los subcomponentes PTR, PC, ME y AgrG fueron de 47, 12,1 y $5 \%$ respectivamente.

\section{Mapas Temáticos de Elementos Paramétricos}

Mapas de aptitud de las tierras. Por clasificación campesina (entrevista a productores) y recorridos en campo para la producción de café (Figura 3a) se encontró que la zona de estudio se puede clasificar en dos clases: una zona "No apta" que se localiza en la parte Noroeste, donde ocurren suelos someros y de textura muy fina denominados por pobladores como "polvillo"; otra zona considerada como "Apta" localizada en la parte sureste, donde reportan suelos profundos con mayor retención de humedad.

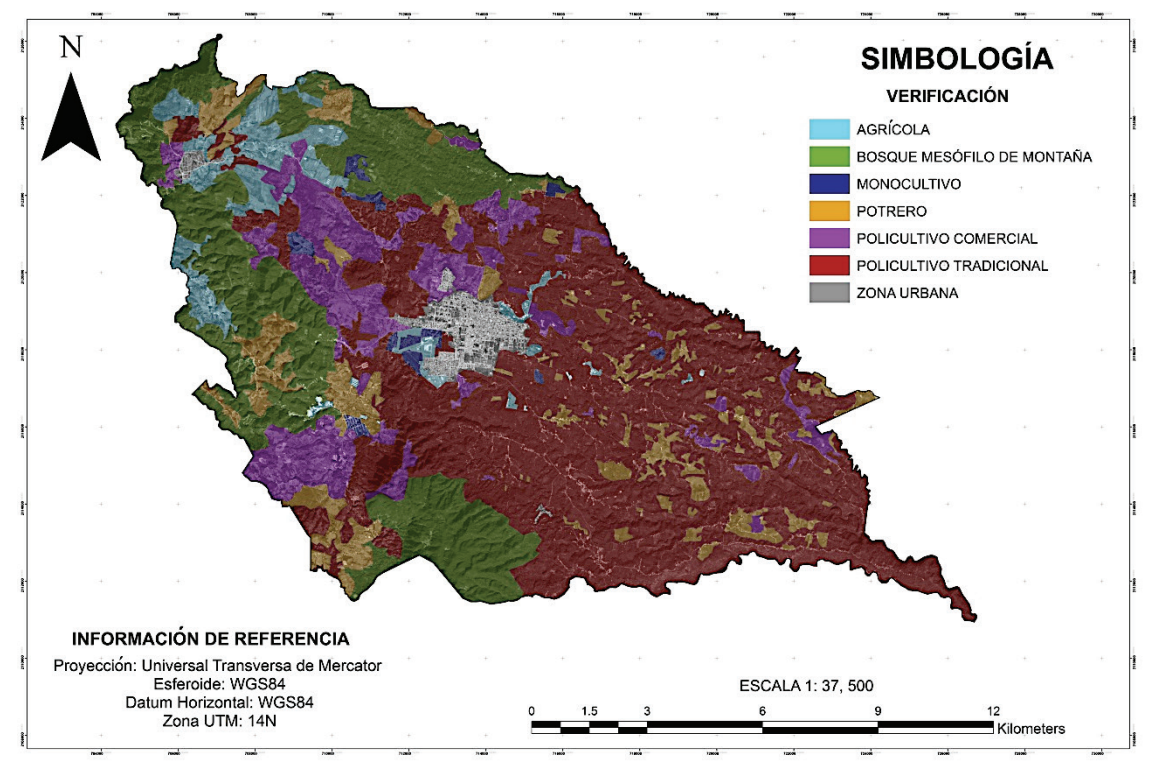

Figura 2. Cobertura de componentes y subcomponentes de los sistemas agroforestales de café a partir del levantamiento fisiográfico con enfoque paisajista. 
Cuadro 1. Áreas de componentes y subcomponentes obtenido en procesos de análisis de imagen.

\begin{tabular}{lc}
\hline Componentes y subcomponentes & Superficie \\
\hline & ha \\
Zona urbana (ZU) & 487.13 \\
Bosque mesófilo de montaña (BMM) & 3753.79 \\
Potrero (Pot) & 1702.93 \\
Agricultura (Agr) & \\
Policultivo tradicional (PT) & 7937.63 \\
Policultivo comercial (PC) & 1999.71 \\
Monocultivo especializado (ME) & 189.56 \\
Agricultura de grano o vegetal (AgrG) & 924.25 \\
\hline
\end{tabular}

El análisis textural de los suelos muestra que los componentes y subcomponentes presentan una clase textural dominante: migajón arenoso $(67 \%$ en BMM); migajón limoso (100\% en Pto), arcillosa (87\% y $82 \%$ en PC y ME respectivamente) y arcillosas y arcillo limosas (50\% cada una en PT). Con base en estos resultados se puede indicar que los suelos de los agrosistemas son predominantemente de textura fina, por lo que tienen una superficie más activa y poseen mayor capacidad de adsorción de nutrimentos en comparación con los suelos arenosos (Ortiz, 2010). En contraste con el bosque mesófilo de montaña que se desarrolla en texturas gruesas y por lo tanto un mejor drenaje.

Mapa de pendiente. Todas las pendientes (Figura 3b) fueron consideradas dentro de la clase "Apta"; no obstante, en el mapa temático se seleccionaron sólo las pendientes por debajo de $25 \%$ que corresponden al $94.7 \%$ de la superficie estudiada y cuyo manejo agronómico es homogéneo (secano). Los valores por grado de pendiente en por ciento fueron obtenidos mediante la reclasificación de un modelo de Elevación Digital (ArcGis ${ }^{\circledR} \mathrm{v} 10.1$ ), y sus superficies estimadas fueron: 0 a $2 \%(28.3 \%), 2-6 \%(29.3 \%), 6-10 \%(19.6 \%)$, $10-15 \%$ (11.1\%), 15-25\% (6.4\%), y $>25 \%$ (5.3\%) del área de estudio. Reagrupando estas clases se tiene que el $77 \%$ de los sistemas agroforestales de café se presentan en pendientes menores de $10 \%$.

Mapa de temperatura. Toda la zona de estudio presenta temperaturas "Aptas" para la producción de café consideración indicada por Pérez et al. (2005). Sin embargo, de acuerdo con los productores y las tres divisiones de calidad por temperatura se logró regionalizar el área de estudio en: alta calidad "ac" $\left(<19{ }^{\circ} \mathrm{C}\right)$ la zona del noroeste con una superficie correspondiente a 19.6\%; mediana calidad "mc" (19 y $22{ }^{\circ} \mathrm{C}$ ), en la zona centro que representa $59.0 \%$ de la superficie y baja calidad "bc" $\left(>22^{\circ} \mathrm{C}\right)$ en el sureste con el $22.4 \%$ del área de estudio, tal cómo se puede observar en la Figura 3c.

Mapa de precipitación. La precipitación fue categorizada como clase "Apta", debido a que en la zona de estudio se presentan precipitaciones entre 1700 y $2000 \mathrm{~mm}$ anuales (SMN, 2017). Este intervalo está dentro de los requerimientos mínimos y máximos reportados por López-Lara et al. (1997) y Benegas $(2009)^{3}$, por lo que no hay restricciones hídricas en la producción de café en la zona de estudio (Figura 3d).

\section{Mapa Temático Compuesto de Sistemas Agroforestales}

Componentes y subcomponentes. El mapa temático de calidad de café (Figura 4) fue obtenido mediante algebra de mapas a partir de los datos paramétricos de los mapas de suelo, pendiente, temperatura y precipitación; posteriormente se re-categorizaron $\mathrm{y}$ se asignaron valores binarios a cada clase ("Cero" o "Uno" a clase "No apta" o "Apta" respectivamente) y criterios como: a) tipo de suelo por aptitud de la tierra; b) grado de pendiente; c) intensidad de precipitación, d) rango de temperatura. Además, se llevó a cabo una re-categorización con sub-índices por calidad de café por la experiencia de los productores y recorridos en campo. Los resultados indican que los sistemas agroforestales tienen diferentes clases y cubren diferentes superficies: a) la clase "ac" de alta calidad se localiza al centro del área de estudio con $25.3 \%$ de la superficie; b) la clase "mc" de mediana calidad se localiza al noroeste y sureste con $33.2 \%$ del área y c) la clase "bc" baja calidad ocurre en la zona centro y noroeste con $45.5 \%$ del área.

Vectorial,polígonos deverificación y comprobación. A partir del mapa temático de agroecosistemas de acuerdo con su aptitud o calidad para la producción de café se obtuvieron 526 polígonos con características particulares entre componentes y subcomponentes; sin embargo, al establecer el criterio cartográfico de área mínima cartografíable (AMC) a escala de trabajo (1:50 000) se re-clasificaron a 111 polígonos con una superficie igual o mayor a 6.25 ha (Figura 5a). En estos polígonos se crearon centroides (Figura 5b) para ser 


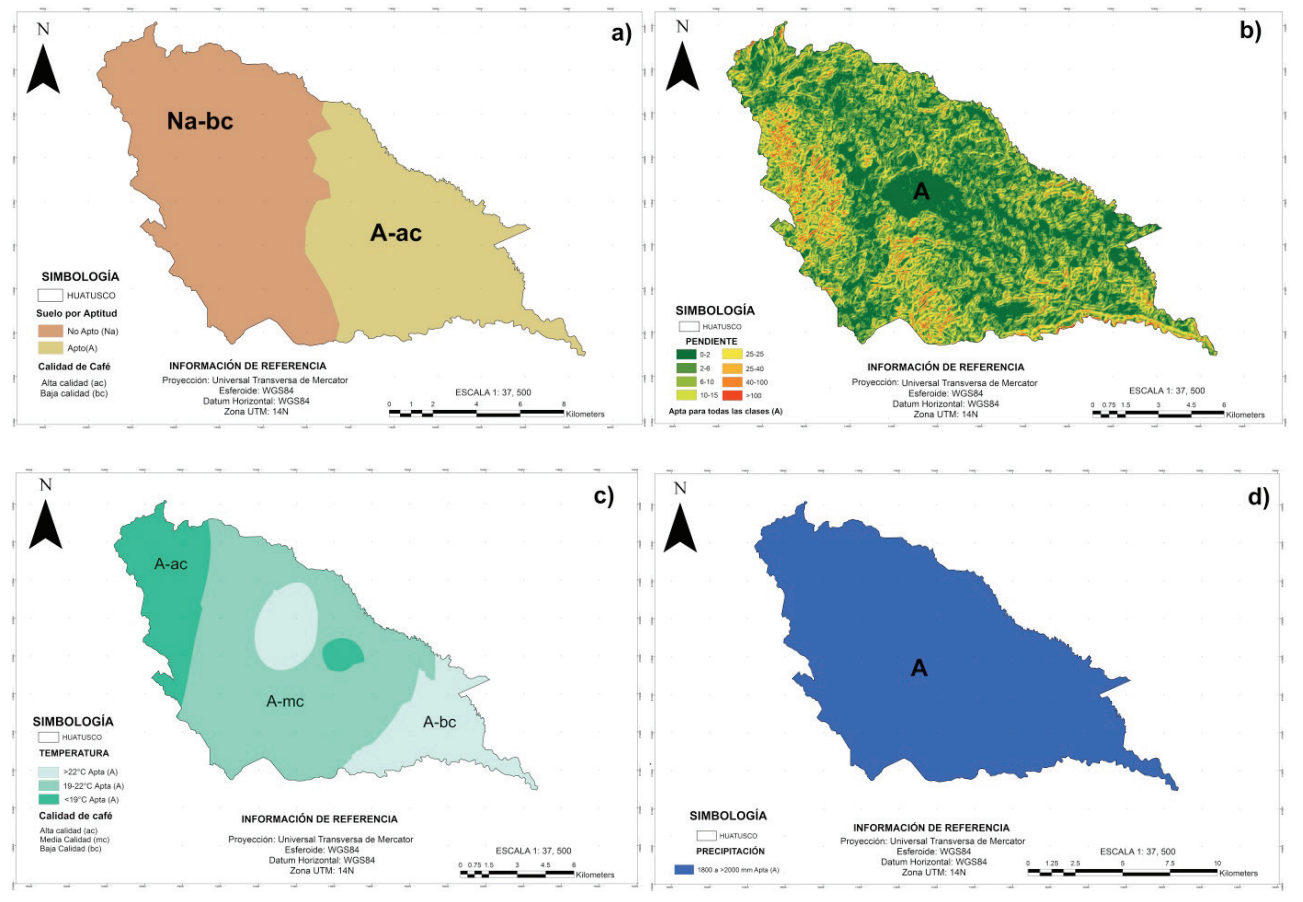

Figura 3. Mapas temáticos de elementos paramétricos. a) Suelo; b) Pendiente; c) Temperatura, y d) Precipitación.

usados como puntos de verificación y comprobación (campos de entrenamiento) por la clasificación interpretativa. Los resultados obtenidos muestran que los componentes y subcomponentes varían en cuanto a su calidad o aptitud para la producción agrícola de café:

a) BMM (68.37 ha). Clase del suelo por aptitud de la tierra "No apta"; pendientes entre 40 y $100 \%$ (No apta); precipitaciones $>1800 \mathrm{~mm}$ anuales; temperatura media anual entre 16 y $20^{\circ} \mathrm{C}$ (apta).

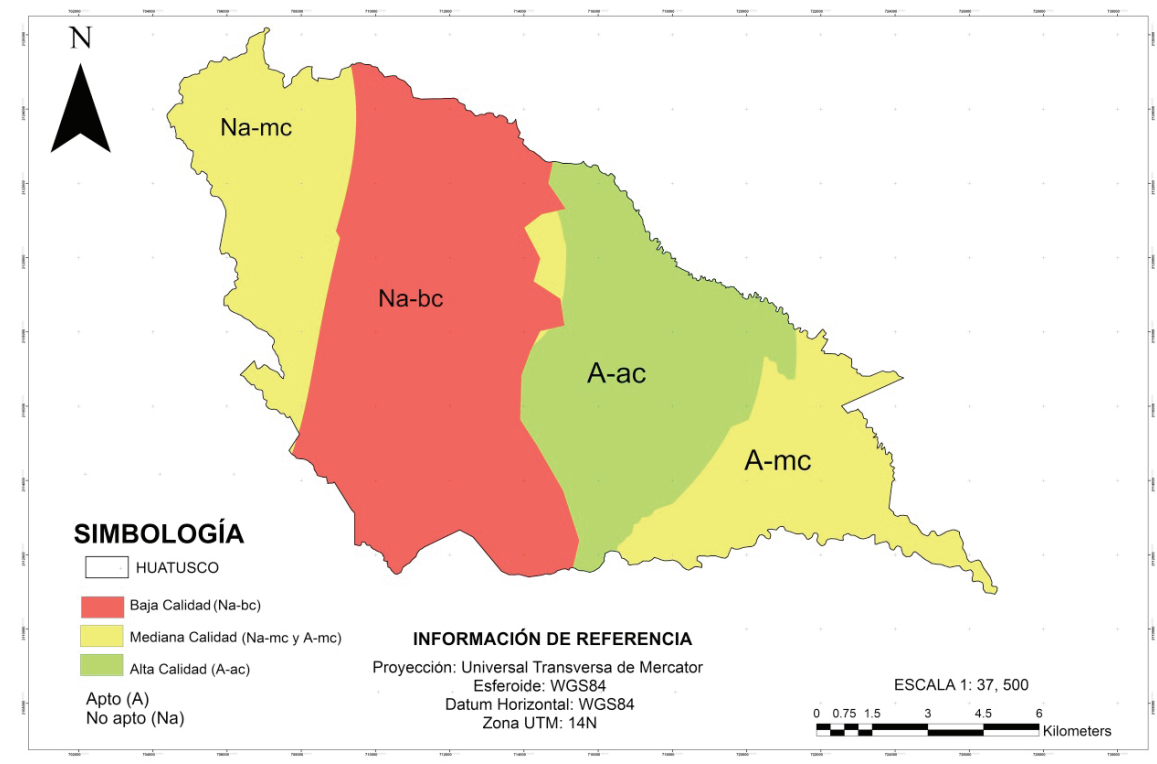

Figura 4. Mapa temático de la aptitud potencial del café, en Huatusco, Veracruz. 
b) Pot (98.21 ha). Clase de suelos por aptitud de la Tierra "No apta"; pendientes $>25 \%$; precipitación media anual $>1800 \mathrm{~mm}$, temperatura media anual $<18^{\circ} \mathrm{C}$.

c) ME (5.81 ha). Clase de suelo por aptitud de la tierra "No Apta"; pendientes $>25 \%$; precipitación media anual $>1800 \mathrm{~mm}$; temperatura media anual entre los 16 y $18{ }^{\circ} \mathrm{C}$;

d) PT, (821.43 ha). Clases de suelo por aptitud de la tierra fueron re-categorizados a 9 sitios como "Aptos" y 37 como "No aptos" (Figura 5b); se presentan dos clases de pendientes: entre $10-15 \%$ y $>25 \%$; precipitación media anual $>1800 \mathrm{~mm}$ y temperatura media anual entre 16 y $20^{\circ} \mathrm{C}$;

e) PC (89.61 ha). Clase de suelo por aptitud de la tierra "No apta" (Figura 5b), debido a que seis sitios presentan pendientes $>40 \%$ y solo uno entre 2 y $4 \%$, precipitación media anual $>1800 \mathrm{~mm}$ anuales y temperatura media anual entre 16 y $20^{\circ} \mathrm{C}$.

Los BMM es un componente que tiene alta biodiversidad (Villaseñor, 2010) y gran variedad de servicios ecosistémicos (Martínez et al., 2009) y ocurre en zonas que fueron clasificadas como no aptas para el cultivo del café. En ese sentido estos resultados pueden ayudar a la toma de decisiones y disminuir la presión del cambio de uso del suelo y evitar su degradación (Orozco et al., 2004). También en los agroecosistemas es necesario evaluar su aptitud de la zona para la producción de café ya que no siempre tienen la calidad y pueden degradar el ambiente, aunque el manejo sea orgánico (Hernández et al., 2017).

\section{Validación de la Clasificación Interpretativa}

La validación se llevó a cabo a partir de recorridos en campo, con apoyo de técnicos de la Coordinación de Fomento Agropecuario, y su comparación sobre el mapa temático de cobertura. La zona de estudio se dividió en cuatro cuadrantes bajo el criterio de accesibilidad y se ubicaron 111 campos de entrenamientos o sitios. Los linderos de cada componente y subcomponentes se modificaron y se georreferenciaron (Figura 5c), además de registrar el tipo de cobertura vegetal agroforestal.

Posteriormente, se seleccionaron 60 sitios como campos de comprobación para generar la matriz de confusión. Los comentarios y experiencias de productores de café definieron el mapa final que pasó de un levantamiento fisiográfico con enfoque paisajista a un mapa de zonificación campesina o conocimiento local sobre la producción de café. La Figura 5d muestra

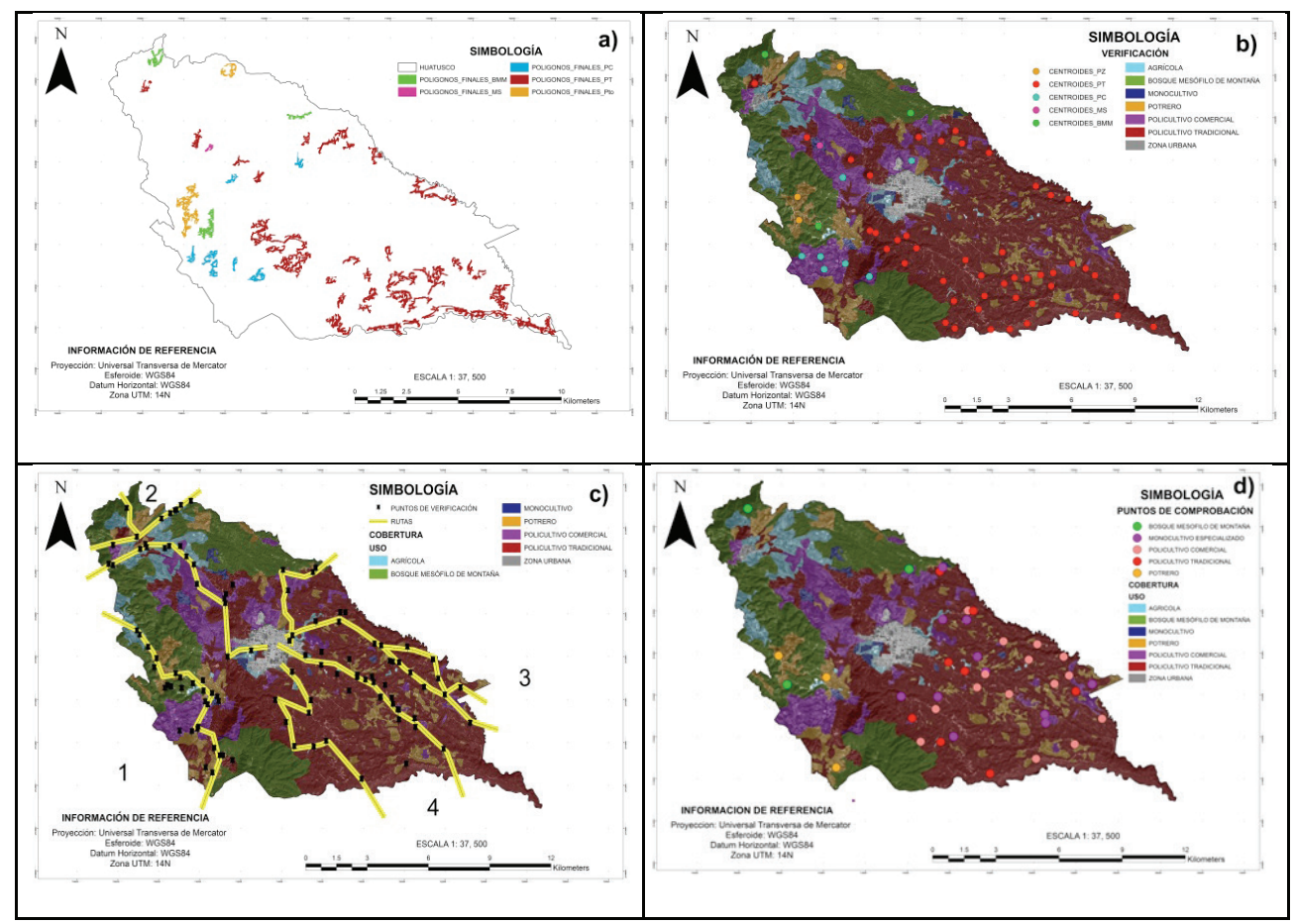

Figura 5. Mapas temáticos de componentes y subcomponentes de los agroecosistemas de café. a) polígonos vectoriales; b) puntos de verificación; c) transectos y recorridos de comprobación, y d) zonificación campesina o conocimiento local y puntos de comprobación. 
los sitios finales de comprobación para cada sistema agroforestal del cultivo de café de los componentes BMM y Pot y sub-componentes PT, PC, ME y AgrG.

Una vez efectuadas las correcciones se generó un mapa temático de cobertura final (Figura 6) que se comparó con el mapa temático de cobertura inicial del levantamiento fisiográfico y mapas temáticos paramétricos (Cuadro 2). Los resultandos indican que se incrementó la superficie con $4.94 \%$ en BMM y con $6.9 \%$ en Pot de acuerdo con la superficie inicial.
En el caso de los subcomponentes se encontró que: a) PT y PC redujeron su superficie en $21.4 \%$ para integrase en una sola cobertura debido a su poca separabilidad entre ellos, b) el ME incrementó en $2.11 \%$, c) la zona agrícola en $0.12 \%$ y una nueva clase denominada Calera no identificada en la fotointerpretación, pero sí en campo la cual fue menor a $0.05 \%$.

La matriz de confusión (Cuadro 3) en sitios de comprobación registró una confiabilidad del productor por cobertura del suelo en PT, PC de 87,

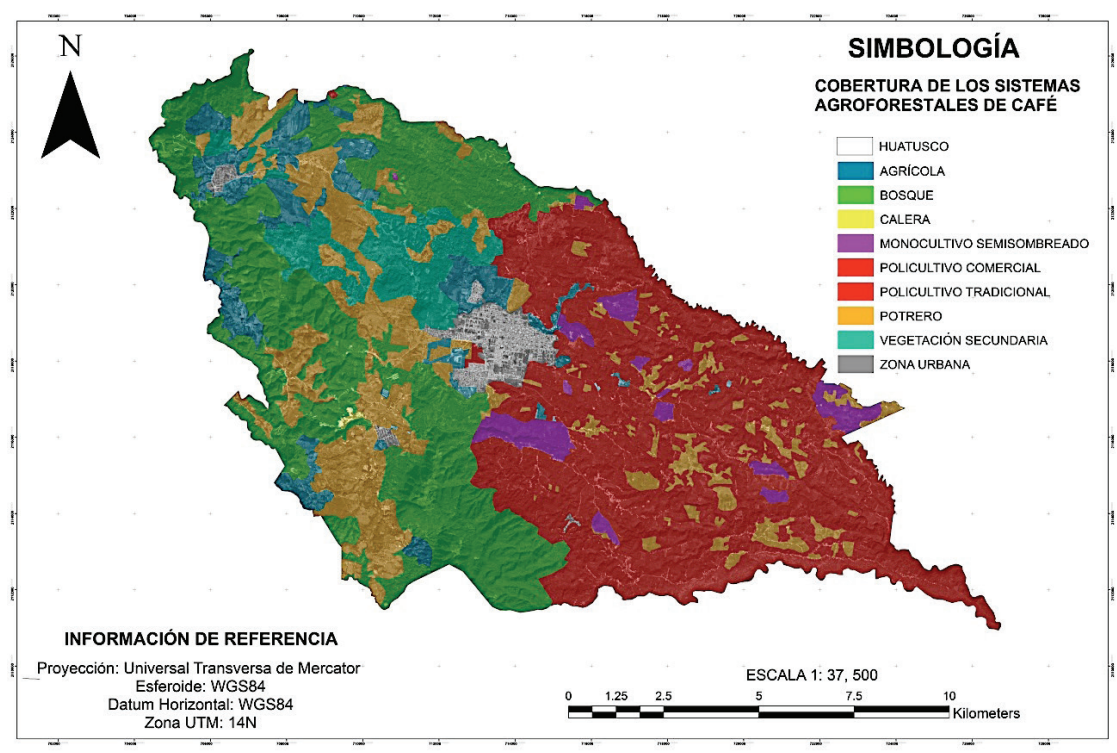

Figura 6. Mapa temático de cobertura de los sistemas agroforestales de café.

Cuadro 2. Comparación de áreas entre componentes y subcomponentes con la verificación de linderos.

\begin{tabular}{|c|c|c|c|c|}
\hline \multirow{2}{*}{ Sistema } & \multicolumn{2}{|c|}{ Cobertura inicial } & \multicolumn{2}{|c|}{ Cobertura final } \\
\hline & Área & Polígonos & Área & Polígonos \\
\hline & ha & & ha & \\
\hline Bosque mesófilo de montaña (BMM) & 3753.79 & 10 & 4593.65 & 18 \\
\hline Policultivo tradicional (PT) & 7937.63 & 111 & 6286 & 109 \\
\hline Policultivo comercial (PC) & 1999.71 & 36 & & \\
\hline Potrero (Pot) & 1702.93 & 96 & 2876.33 & 116 \\
\hline Agrícola (Agr) & 924.25 & 26 & 1194.69 & 32 \\
\hline Zona urbana & 487.13 & 4 & 508.33 & 5 \\
\hline Monocultivo especializado (ME) & 189.56 & 11 & 548.63 & 17 \\
\hline Vegetación secundaria (VS) & - & - & 972.09 & 14 \\
\hline Calera & - & - & 14.89 & 1 \\
\hline Suma & 16995.00 & 294 & 16995.00 & 312 \\
\hline
\end{tabular}


Cuadro 3. Matriz de confusión de puntos de comprobación de componentes y subcomponentes en los sistemas agroforestales de café.

\begin{tabular}{|c|c|c|c|c|c|c|c|c|c|}
\hline \multicolumn{2}{|c|}{ Componente subcomponente } & \multirow{2}{*}{$\frac{\text { BMM }}{3}$} & \multirow[t]{2}{*}{ Pot } & \multirow[t]{2}{*}{ ME } & \multirow[t]{2}{*}{ PT } & \multirow[t]{2}{*}{$\mathrm{PC}$} & \multirow[t]{2}{*}{ Otro } & \multirow[t]{2}{*}{ Total } & \multirow{2}{*}{$\begin{array}{c}\begin{array}{l}\text { Confiabilidac } \\
\text { del usuario }\end{array} \\
1\end{array}$} \\
\hline \multirow{7}{*}{ 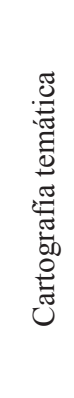 } & BMM & & & & & & & & \\
\hline & Pot & & 3 & & & & & & 1 \\
\hline & ME & & & 1 & & & & & 1 \\
\hline & PT & & & & 21 & 15 & 4 & 46 & 0.46 \\
\hline & $\mathrm{PC}$ & & & & & 4 & & & 0.57 \\
\hline & Otro & & & & & & & & \\
\hline & Total & 3 & 3 & 1 & 24 & 19 & 4 & 57 & \\
\hline \multicolumn{2}{|c|}{ Confiabilidad del productor } & 1 & 1 & 1 & 0.87 & 0.79 & 1 & & \\
\hline
\end{tabular}

$\mathrm{BMM}=$ bosque mesófilo de montaña $\mathrm{Po}=$ potreros; $\mathrm{Me}=$ monocultivo especializado $\mathrm{Pt}=$ policultivo tradicional $; \mathrm{Pc}=$ policultivo comercial.

$79 \%$ respectivamente y de $100 \%$ en BMM, Pot y ME. La matriz de confusión o de contingencias, consta de una tabla de doble entrada, que confronta los valores reales o verdad- terreno con los resultados de la clasificación (Cohen, 1960). De acuerdo con Bishop et al. (1975), valores mayores de $70 \%$ ya son aceptables en cuanto a la calidad de los mapas. En ese sentido, la información proporcionada por los productores incrementó la precisión de los mapas temáticos hasta prácticamente presentar alta concordancia (100\%).

\section{Información Adicional}

Los productores reportaron que las especies vegetativas exóticas más usadas como sombra en los plantíos de cafetos de la región son: coquito plumoso (Syagrus romanzoffiana), primavera (Tabebuiadonnellsmithii Rose), cedro rosado (Acrocarpus fraxinifolius), mango piña, palma criollito, cedro rojo (Cedrela odorata L.), cacao (Theobroma cacao L.), aguacate, chalahuite (Inga spuria Humb. \& Bonpl. ex Willd), naranjo, vainillo de hoja ancha (Vanilla planifolia), macadamia (Macadamia spp.), chichene, palma, plátano tabasco, fresno (Fraxinus uhdei), tinaja, malta, roatán, brebil, jinicuil, ixpepe (Trema micranthum), sopa de pan, luminaria, pimienta y zapote (Pouteria sapota). Además, también reportaron especies vegetales nativas en el componente BMM como: guayabillo (Alibertia edulis), encino rojo (Quercus rubra Née), encino blanco (Quercus sartorii), roble, liana, y aguacatillo lo que provocó la homogeneización visual sobre las imágenes satelitales. Esta es una evidencia de la alta biodiversidad de estos agroescosistemas como ha sido reportado por otros autores (Moguel y Toledo, 1999; Perfecto y Armbrecht, 2003; Escamilla et al., 2005).

\section{CONCLUSIONES}

- La fotointerpretación y clasificación interpretativa permitió clasificar dos componentes agroforestales: bosque mesófilo de montaña y potreros y tres subcomponentes agrícolas: policultivo tradicional $\mathrm{y}$ comercial, monocultivo especializado, identificados a través de su brillantez y textura en una imagen de satélite. Sin embargo, en el componente agrícola hay confusión en las estructuras vegetativas nativas y comercial. Con la participación de los productores (entrevistas, campos de entrenamiento y recorridos de campo) y mapas temáticos de elementos paramétricos se identificaron con una precisión de 100\% los bosques, los potreros y el monocultivo especializado.

- La zona de mayor aptitud de las tierras para la producción de café se localiza en la zona centro; mientras que los de baja calidad en el centro y noreste del municipio de Huatusco en donde ocurren los bosques mesófilos de montaña o policultivos tradicionales y comerciales.

- La combinación entre técnicas geoespaciales con el conocimiento local de los productores permite identificar zonas con potencial productivo agrícola de café y dar elementos para la conservación de otros ambientes como los bosques mesófilos de montaña. 


\section{LITERATURA CITADA}

Anaya Garduño, M. 1991. Manual de conservación del suelo y del agua. Colegio de Postgraduados, Chapingo, SARH, SPP. Chapingo. Edo. de México, México.

Bishop, Y. M. M., S. E. Feinberg, P. W. Holland, R. J. Light, and F. Mosteller. 1975. Discrete multivariate analysis: Theory and practice. The Mit Press. Cambridge, MA, USA.

Bolstad, P. 2012. GIS fundamentals. A first text on geographic information systems. Eider Press. ISBN 978-0-9717647-3-6.

Borrás, J., J. Delegido, A. Pezzola, M. Pereira, G. Morassi, and G. Camps-Valls. 2017. Land use classification from sentinel-2 imagery. Rev. Teledetección 48: 55-66. doi: 10.4995/ raet.2017.7133.

Brody, R. 1999. Geographic information systems. J. Busin. Finan. Librariansh. 5: 3-18. doi:10.1300/J109v05n01 02.

Buendía R., E., S. Terrazas D. y E. Vargas P. 2002. Aplicación de imágenes de satélite en la cartografía de uso de suelo y vegetación en una región del oriente del Valle de México. Rev. Chapingo Serie Cienc. For. Amb. 8: 13-19.

Cayssials, R. y C. Alvarez. 1983. Interpretación agronómica de la carta de reconocimiento de suelos del Uruguay. Boletín técnico No. 9. MGAP, DGRNR dirección de suelos. Montevideo, Uruguay.

Chuvieco, S. E. 2008. Teledetección ambiental. La observación de la tierra desde el espacio. Ariel. Barcelona, España. ISBN-10: 8434434989.

Cohen, J. 1960. A coefficient of agreement for nominal scales. Educat. Psychol. Measur. 20: 37-46.

ECOCROP (Crop Ecological Requirements Database). 2018. Crop Ecological Requirements Database. FAO - Organización de las Naciones Unidas para la Alimentación y la Agricultura. http:// ecocrop.fao.org/ecocrop/srv/en/home. (Consulta: mayo 20, 2018).

Escamilla P., E. y S. Díaz C. 2002. Sistema de cultivo de café en México. Universidad Autónoma Chapingo. Huatusco, Veracruz, México.

Escamilla P., E., O. Ruiz R., G. Diaz P., C. Landeros S., D. E. Platas R., A. Zamarripa C. y V. A. González H. 2005. El agroecosistema café orgánico en México. Manejo Integr. Plagas Agroecol. (Costa Rica) 76: 5-16.

García M., E. 2006. Análisis visual de imágenes. Área de Geodinámica Externa. Depto. de Geología. Universidad de León. León, España.

Gómez T., A., Y. Chávez H., L. M. Morales M., E. Díaz F., A. F. Gómez, T. R. y H. Zepeda C. 2003. Predicción espacial de carbono edáfico superficial en zonas forestales mediante análisis digital de terreno y sig: Uso de la radiación solar potencial. For. Veracruz. 5: 49-56.

Guerra Martínez, V. y S. Ochoa Gaona. 2005. Identificación y variación de la vegetación y uso del suelo en la reserva pantanos de Centla, Tabasco (1990-2000) mediante sensores remotos y sistemas de información geográfica. Ra Ximhai 1: 325-346.

Gutiérrez P., J y M. Gould. 1994. SIG: Sistemas de información geográfica. Ed. Síntesis. Madrid, España. ISBN: 9788477382461.
Hernández Ordoñez, L. O., M. del C. Gutiérrez Castorena, C. A. Ortiz Solorio, P. Sánchez Guzmán y E. Ángeles Cervantes. 2017. Calidad de los Andosols en sistemas forestal, agroforestal y agrícola en diferentes manejos en Zacatlan, Puebla. Terra Latinoamericana 35: 179-189.

Instituto de Investigación de Recursos Biológicos Alexander Von Humboldt. 2006. Los sistemas de información geográfica. Geoenseñanza 11: 107-116.

INEGI (Instituto Nacional de Estadística y Geografía). 2009a. Conjunto Edafología. Conjunto de datos vectoriales Edafología Serie I. Aguascalientes, México.

INEGI (Instituto Nacional de Estadística y Geografía). 2009b. Prontuario de Información Geográfica Municipal de los Estados Unidos Mexicanos, Huatusco. Clave geoestadística 21053 y 21208. Documento en línea en http://mapserver. inegi.org.mx/dsist/prontuario/index2.cfm. (Consulta: abril 10, 2015).

Lencinas, J. D. 2011. Tecnología geomática para la evaluación de bosques nativos en Patagonia. CIEFAP. Esquel, Chubut, Argentina.

López-Lara, E. J., J. C. Posada S., J. G. Moreno N. 1997. Los sistemas de información geográfica. pp. 789-804. In: J. Rodríguez García y J. C. Collado Machuca (eds.). Andalucia en el umbral del siglo XXI. I Congreso de Ciencia Regional de Andalucia. ISBN/ISSN: 84-7786-534-5.

Martínez, M. L., O. Pérez-Maqueo, G. Vázquez, G. Castillo Campos, J. Garcia-Franco, K. Mehltreter, M. Equihua, and R. Landgrave. 2009. Effect of land use change on biodiversity and ecosystem services in tropical montane cloud forest of Mexico. For. Ecol. Manage. 258: 1856-1863. doi: 10.1016/j. foreco.2009.02.023.

Moguel, P. y V. M. Toledo. 2004. Conservar produciendo: Biodiversidad, café orgánico y jardines productivos. Biodiversitas 55: 2-7.

Orozco, H. E., V. Peña, R. Franco y N. Pineda. 2004. Altlas agrario ejidal del Estado de México. Cuadernos de Investigación Núm. 34. UAEM. Toluca, Estado de México, México.

Orradre, M. N. 2014. Métodos de clasificación de imágenes satelitales para la determinación de usos del suelo agrícola en el partido de Tandil (Provincia de Buenos Aires, Argentina). Geogr. Sist. Inf. Geográf. 6: 11-28.

Ortiz S., C. A. 2010. Edafología. Universidad Autónoma Chapingo. Texcoco, México.

Ortiz S., C. y H. E. Cuanalo. 1978. Metodología del levantamiento fisiográfico: un sistema de clasificación de tierras. Colegio de Postgraduados. Chapingo, México.

Ortiz S., C. A. y M. C. Gutiérrez C. 1999. Evaluación taxonómica de sistemas locales de clasificación de tierras. Terra 17: 277-286.

Pérez P., E., J. G. Partida S. y D. Martínez P. 2005. Determinación de las subdenominaciones de origen del café Veracruz (estudio preliminar). Rev. Geogr. Agríc. 35: 23-56.

Perfecto, I. and I. Armbrecht. 2003. The coffee agroecosystem in the neotropics: Combining ecological and economic goals. Chapter 6. pp.159-194. In: J. H. Vandermeer (ed.). Tropical agroecosystems. CRC Press. Boca Raton, FL, USA. ISBN: 0-8493-1581-6.

Perfecto, I., J. Vandermeer, A. Mas, and L. Soto P. 2005. Biodiversity, yield, and shade coffee certification. Ecol. Econ. 54: 435-446. doi: 10.1016/j.ecolecon.2004.10.009. 
Portuguez Yactayo, H. 1998. Mapa de bosques secos del departamento de Piura. INR, DGEP. Lima, Perú.

Reyes-García, V. 2009. Conocimiento ecológico tradicional para la conservación: Dinámicas y conflictos. Papeles 107: 39-55.

Rosete, F. y G. Bocco. 2003. Los sistemas de información geográfica y percepción remota. Herramientas integradas para los planes de manejo en comunidades forestales. Gaceta Ecol. 68: $43-54$

Rullán S., C. D., L. M. Gama C., A. Galindo A. y A. E. Olthoff. 2011. Clasificación no supervisada de la cobertura de suelo de la región Sierra de Tabasco mediante imágenes LANDSAT ETM+. Univ. Cienc. 27: 33-41.

SMN (Servicio Meteorológico Nacional). 2017. Normales climáticas (1951-2010). http://smn.cna.gob.mx/es/ climatológia/información-climatologica. (Consulta: marzo 15, 2017).
Vega G., Á., J. López G. y L. L. Manzo D. 2008. Análisis espectral y visual de vegetación y uso del suelo con imágenes Landsat $\mathrm{ETM}+$ con apoyo de fotografías aéreas digitales en el Corredor Biológico Chichinautzin, Morelos, México. Invest. Geogr 67: 59-75.

Verdú, F. y J. Salas R. 2010. Cartografía de áreas quemadas mediante análisis visual de imágenes de satélite en la España peninsular para el periodo 1991-2005. Rev. Int. Cienc. Tecnol. Inf. Geográf. 10: 54-81.

Villaseñor, J. L. 2010. El bosque húmedo de montaña en México y sus plantas vasculares: Catálogo florístico-taxonómico. Comisón Nacional para el Conocimiento y Uso de la Biodiversidad-UNAM. México. D. F. ISBN: 978-607-021557-5.

Zorogastúa C., P., R. Quiroz G. y J. Garatuza P. 2011. Evaluación de cambios en la cobertura y uso de la tierra con imágenes de satélite en Piura-Perú. Ecol. Aplic. 10: 13-22. 